\title{
Analysis of Solid-Liquid Interface Behavior during Continuous Strip-Casting Process Using Sharp-Interface Technique
}

\author{
Changbum Lee, ${ }^{1}$ Wooyoung Yoon, ${ }^{1}$ Seungwon Shin, ${ }^{2}$ Jaewoo Lee, ${ }^{1}$ and Hee-eun Song ${ }^{3}$ \\ ${ }^{1}$ Materials Science and Engineering, Korea University, Seongbuk-gu, Seoul 136-713, Republic of Korea \\ ${ }^{2}$ Department of Mechanical and System Design Engineering, Hongik University, Seoul 127-791, Republic of Korea \\ ${ }^{3}$ Solar Energy Research Center, Korea Institute of Energy Research, 152 Gajeong-ro, Yuseong-gu, \\ Daejeon 305-343, Republic of Korea
}

Correspondence should be addressed to Wooyoung Yoon; alloylab@gmail.com

Received 31 January 2013; Accepted 2 May 2013

Academic Editor: Donghwan Kim

Copyright (c) 2013 Changbum Lee et al. This is an open access article distributed under the Creative Commons Attribution License, which permits unrestricted use, distribution, and reproduction in any medium, provided the original work is properly cited.

Continuous strip casting (CSC) has been developed to fabricate thin metal plates while simultaneously controlling the microstructure of the product. A numerical analysis to understand the solid-liquid interface behaviors during CSC was carried out and used to identify the solidification morphologies of the plate, which were then used to obtain the optimum process conditions. In this study, we used a modified level contour reconstruction method and the sharp-interface method to modify the interface tracking, and we performed a simulation analysis to identify the differences in the material properties that affect the interface behavior. The effects of the process parameters such as the heat transfer coefficient and extrusion velocity on the behavior of the solid-liquid interface are estimated and also used to improve the CSC process.

\section{Introduction}

Identifying the underlying mechanisms that occur during the solidification process is essential for determining the microstructure of a material, which in turn determines the physical properties of the final product. Understanding the solid-liquid interface system is important for understanding the morphology, but the dynamics of an interface are relatively complex, both experimentally and theoretically, even for the simplest substances $[1,2]$. Continuous strip casting (CSC) is a horizontal continuous-casting technique designed to yield a thin metal plate or strip while simultaneously controlling the microstructure. CSC is also a promising technique for producing silicon wafers without kerf loss for silicon solar cells [3]. Because the size and orientation of the silicon grains significantly affect the solar cell efficiency [4], CSC could be used to fabricate thin Si strips. A planar solidliquid interface needs to have a longitudinal grain structure, and therefore, the simulation model for CSC should take into account the interface behaviors. In a previous study, tracking of a solid-liquid interface was performed with numerical simulation of the CSC process [3]. In this paper, we studied the solid-liquid interface behavior as a function of the material properties using the level contour reconstruction method (LCRM). Interface tracking and the analysis of the shape of the solid-liquid interface with a specific heat transfer coefficient were carried out in order to determine the optimum CSC process parameters. The sharp-interface method employed in a few earlier studies [5-7] was also used in this study to implement the exact boundary conditions for a moving solid interface, thus maintaining an accurate phase equilibrium at the solid-liquid interface and enabling us to perform a simulation analysis of the CSC process. Front tracking, the method used here, has many advantages, including its lack of numerical diffusion and the ease and accuracy with which interfacial physics can be described on a subgrid level. It was found that tracking often does not require highly refined grids and that the grid orientation does not affect the numerical solution [8]. Tracking affords a precise description of the location and geometry of the interface, and thus, the surface tension force can be very accurately computed directly on the interface [9]. 


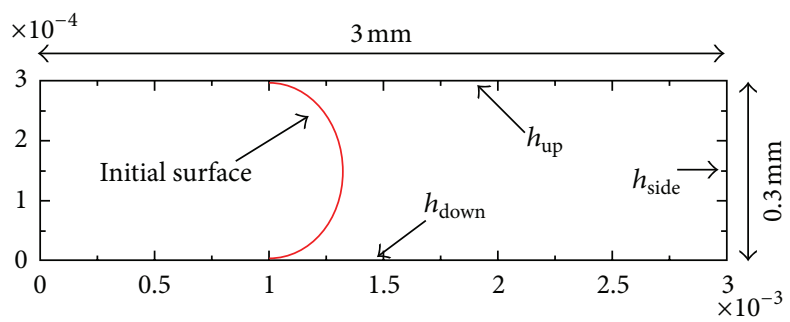

FIgURE 1: Dimensions $(3 \times 0.3 \mathrm{~mm})$ for the numerical simulation. Initial interface and heat extraction sides are marked $\left(h_{\mathrm{up}}, h_{\mathrm{down}}\right.$, and $\left.h_{\text {side }}\right)$.

\section{Materials and Methods}

A schematic of the equipment apparatus, designed for manufacturing a continuous thin metal plate, is shown in Figure 1. Two heating zones were installed: one to control the melt temperature and the other to apply a temperature gradient. A cooling system was used to extract heat from the liquid for solidification. The pressure, which determines the production rate of the metal plate, was controlled by a mass flow controller (MFC) and a vent valve. The dummy bar was used as a stopper and the heat extraction route. A numerical simulation was performed for optimizing the continuous casting parameters. The single-field formulation of the continuity and momentum equation is solved for incompressible twophase flow:

$$
\begin{gathered}
\nabla \cdot u=0 \\
\rho\left(\frac{\partial u}{\partial t}+u \cdot \nabla u\right)=-\nabla P+\rho g+\nabla \cdot \mu\left(\nabla u+\nabla u^{T}\right)+F \\
\frac{\partial T}{\partial t}+u \cdot \nabla T=\alpha \nabla^{2} T\left(\begin{array}{c}
\alpha=\alpha_{L} \text { in liquid phase } \\
\alpha=\alpha_{S} \text { in solid phase }
\end{array}\right)
\end{gathered}
$$

where $u$ is the velocity, $P$ is the pressure, $g$ is the gravitational acceleration, and $F$ is the local surface tension force at the interface. Material property fields can be described by using the indicator function $I(x, t)$. The density is calculated by

$$
\rho(x, t)=\rho_{1}+\left(\rho_{2}-\rho_{1}\right) I(x, t) .
$$

The interface is advected in a Lagrangian fashion by integrating

$$
\frac{d x_{f}}{d t} \cdot n=\frac{\dot{m}_{f}}{\rho_{f}}=V \cdot n
$$

where $V$ is the interface velocity vector. In problems involving phase changes, the interface velocity and the fluid velocity at the interface are not necessarily the same. Without a phase change, the interface velocity will be equal to the fluid velocity at the interface, that is, $V=u f=u(x f)$. Only the normal component of the interface motion is determined by the physics and not the tangential motion, and we may assume that the interface and fluid at the interface have the same tangential component of velocity.
The equation of conservation of mass written for the entire flow field is

$$
\frac{\partial \rho}{\partial t}+\nabla \cdot(\rho u)=0
$$

The momentum equation is written for the entire flow field and the forces resulting from surface tension and inserted at the interface as body forces, which act only at the interface. In conservative form, this equation is

$$
\begin{aligned}
\frac{\partial(\rho u)}{\partial t}+\nabla \cdot(\rho u u)= & -\nabla P+\rho g+\nabla \cdot \mu\left(\nabla u+\nabla u^{T}\right) \\
& +\int_{\Gamma(t)} \sigma \kappa n \delta\left(x-x_{f}\right) d s,
\end{aligned}
$$

where $\sigma$ is the surface tension coefficient and $\kappa$ is twice the mean interface curvature. The thermal energy equation with an interfacial source term to account for the liberation or absorption of latent heat is

$$
\frac{\partial(\rho c T)}{\partial t}+\nabla \cdot(\rho u c T)=\nabla \cdot k \nabla T+\int_{\Gamma(t)} \dot{m}_{f} L \delta\left(x-x_{f}\right) d s
$$

Here, $T$ is the temperature and $L=L_{0}+\left(c_{1}-c_{2}\right) T_{\text {sat }} \cdot L_{0}$ is the latent heat measured at the equilibrium saturation temperature $T_{\text {sat }}(P)$, corresponding to the reference ambient system pressure. The Navier-Stokes equation is derived by the phase-change projection method [10] using firstorder forward Euler time integration [11]. In this study, the marker-and-cell method [12] is used for staggered grid discretization. The convection term, viscosity term, and pressure field are calculated by the second-order essentially nonoscillatory (ENO) method, the central difference method, and FISHPACK [13], respectively. The detailed procedures for solving the governing equations and introducing the interface-tracking method can be found in $[9,10,14,15]$. In front tracking, the customary stationary volumetric mesh is supplemented by a moving interface mesh that is used to explicitly track the interface. This interface mesh is composed of nonstationary Lagrangian computational points connected to form a two-dimensional surface. At each time step, information must be passed between the moving Lagrangian interface and the stationary Eulerian grid. Because the Lagrangian interface points do not necessarily coincide with the Eulerian grid points, the passing of information is accomplished using Peskin's immersed boundary method [15]. With this technique, an infinitely thin interface is approximated by a smooth distribution function that is used to distribute sources at the interface over several grid points near the interface. In a similar manner, this function is used to interpolate field variables from the stationary grid to the interface. In this way, the front is given a finite thickness on the order of the mesh size to provide stability and smoothness. There is also no numerical diffusion because this thickness remains constant. Our LCRM [14] is a simplified front-tracking method that eliminates the logical connectivity between discrete interface elements. The associated algorithmic and 


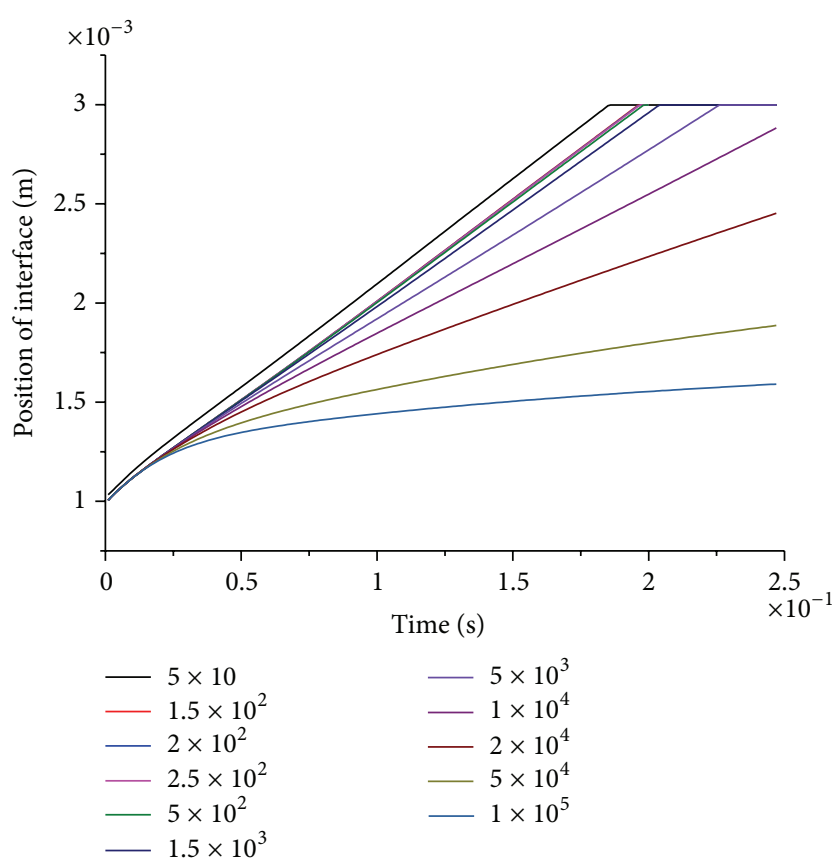

FIGURE 2: Numerical results of the location of the silicon solid-liquid interface with time at a fixed external velocity $(0.01 \mathrm{~m} / \mathrm{s})$. The heat transfer coefficient ranged from $50 \mathrm{~W} / \mathrm{m}^{2} \mathrm{~K}$ to $1.0 \times 10^{5} \mathrm{~W} / \mathrm{m}^{2} \mathrm{~K}$.

bookkeeping burden is eliminated, and at the same time, the accuracy and advantages of explicit Lagrangian surface tracking are retained. The interface elements are periodically discarded and then reconstructed on a level contour of the characteristic indicator function $I(x, t)$. The newly reconstructed interface elements automatically take on the topological characteristics of the indicator function, and thus, the operations of element deletion, addition, and reconnection are accomplished simultaneously and automatically in one step, without the need for element connectivity. Furthermore, once the elements are constructed, the interface normal and element areas are easily defined and surface tension forces are accurately computed directly on the interface for each element independently.

\section{Results and Discussion}

The dimensions of the simulated model are shown in Figure 1. The initial surface is assumed to be a semicircular shape, and the heat extraction of both the up side and the down side was assumed to be the same. The range of heat extraction applied was from $50 \mathrm{~W} / \mathrm{m}^{2} \mathrm{~K}$ to $1.0 \times 10^{5} \mathrm{~W} / \mathrm{m}^{2} \mathrm{~K}$, and the range of the external velocity $\left(V_{\text {ex }}\right)$ was from $0.001 \mathrm{~m} / \mathrm{s}$ to $1 \mathrm{~m} / \mathrm{s}$. To analyze the behavior of the solid-liquid interface, we tracked the location of the interface and the temperature change. The importance of the final solid-liquid region has been studied before [3]; therefore, the position of the interface should be analyzed in order to understand the behavior. The location of the solid-liquid interface as a function of time at an external velocity of $0.01 \mathrm{~m} / \mathrm{s}$ is shown in Figures 2 and 3 for silicon and tin, respectively. The heat transfer coefficient, which denotes

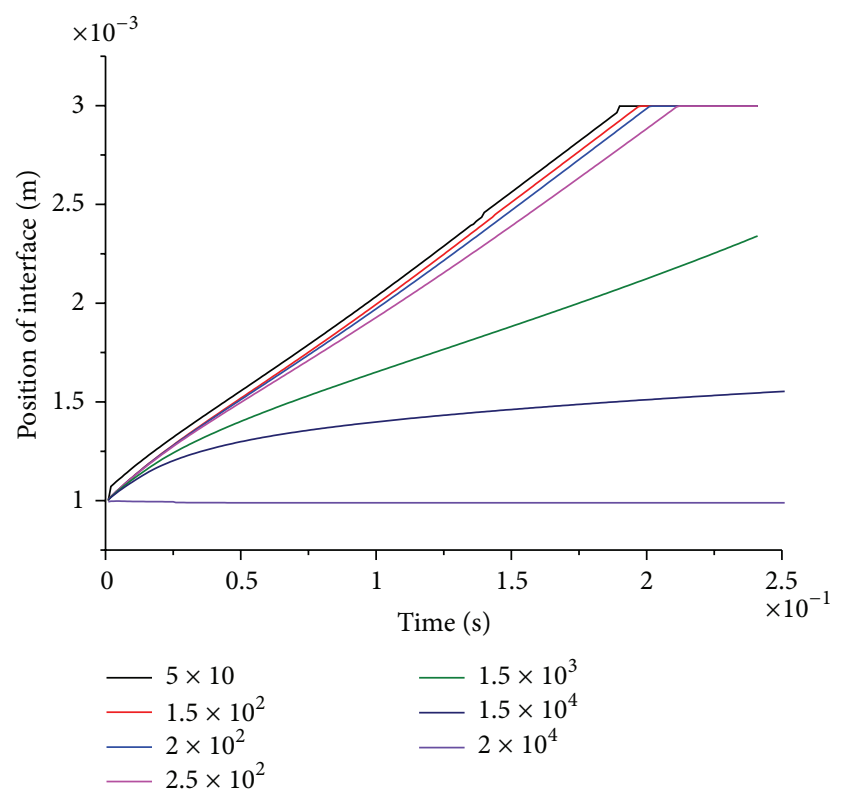

FIGURE 3: Numerical results of the location of the tin solid-liquid interface with time at a fixed external velocity $(0.01 \mathrm{~m} / \mathrm{s})$. The heat transfer coefficient ranged from $50 \mathrm{~W} / \mathrm{m}^{2} \mathrm{~K}$ to $2.0 \times 104 \mathrm{~W} / \mathrm{m}^{2} \mathrm{~K}$.

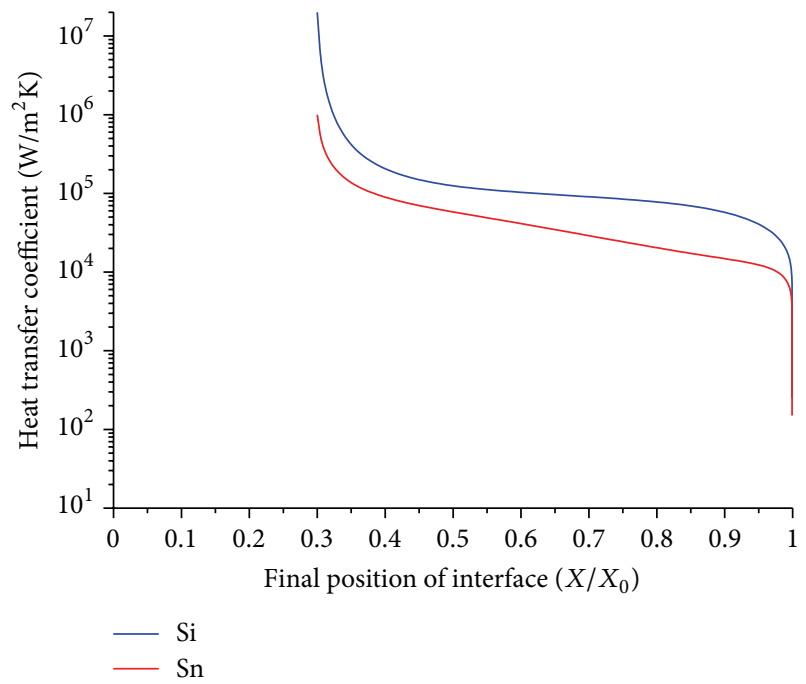

FIGURE 4: Final location of the solid-liquid interface with each heat transfer coefficient at a fixed external velocity $(0.01 \mathrm{~m} / \mathrm{s})$.

the amount of heat transferred from a liquid to a mold and solid, ranged from $50 \mathrm{~W} / \mathrm{m}^{2} \mathrm{~K}$ to $1.0 \times 10^{5} \mathrm{~W} / \mathrm{m}^{2} \mathrm{~K}$.

As shown in Figure 4, the locations of the final solidliquid interfaces are classified according to the heat transfer coefficients: $1.2 \times 10^{3}$ to $1 \times 10^{6}, 9 \times 10^{2}$ to $1.2 \times 10^{3}$, and below $9 \times 10^{2} \mathrm{~W} / \mathrm{m}^{2} \mathrm{~K}$ for tin and silicon. In particular, the position of the interface is moved away from the boundary when the heat transfer coefficient is less than $9 \times 10^{2} \mathrm{~W} / \mathrm{m}^{2} \mathrm{~K}$ with a $0.01 \mathrm{~m} / \mathrm{s}$ extrusion velocity. The interface is not positioned in the chamber at small values of the heat transfer coefficient, and the interfaces at heat transfer coefficients of $1.2 \times 10^{3}$ 
TABLE 1: The temperature mapping of simulation dimension of each heat extrude direction and heat transfer coefficient at final state.

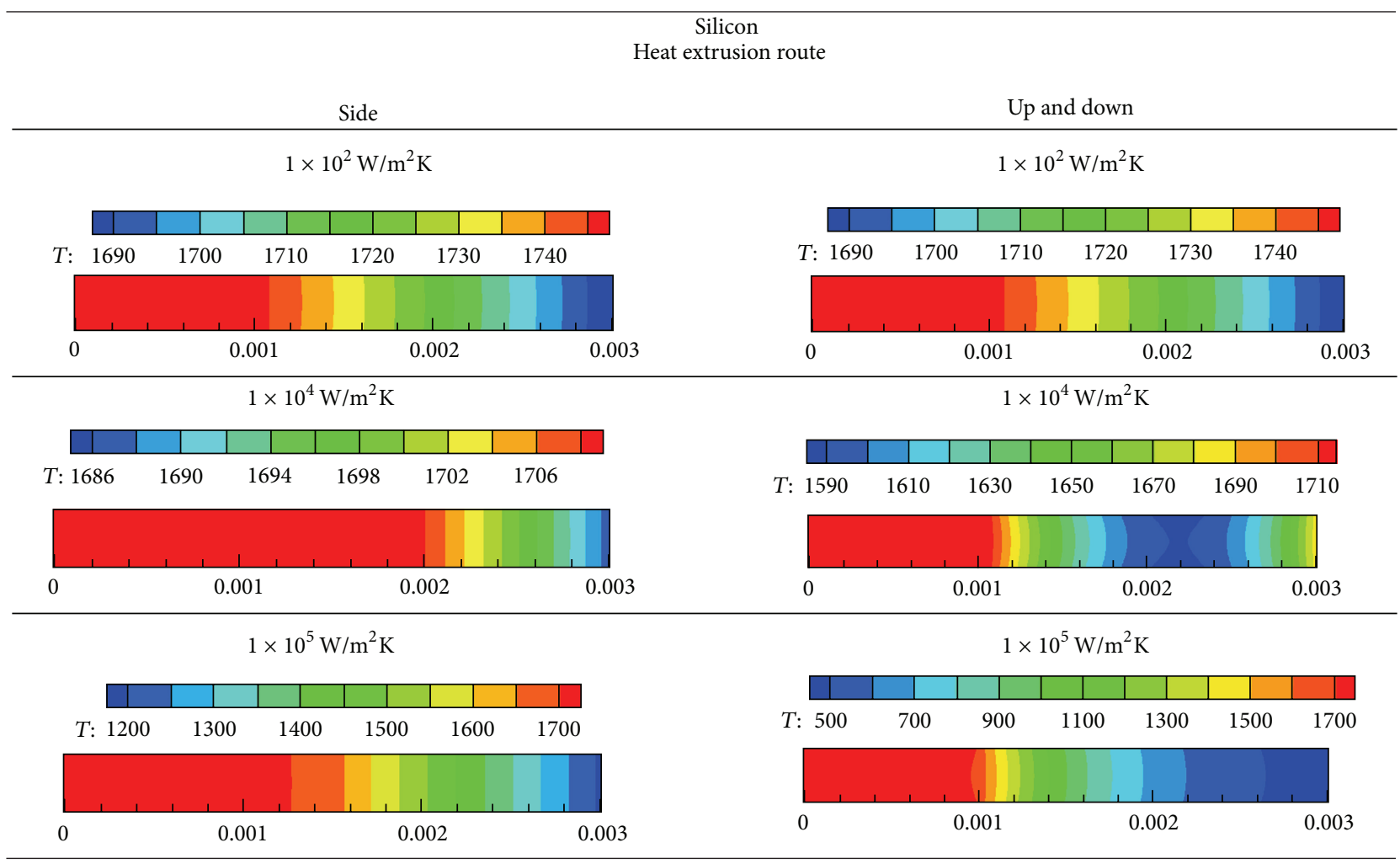

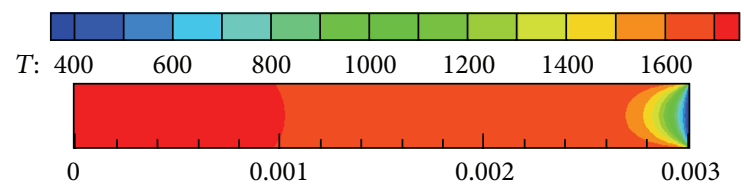

FIgURE 5: Temperature mapping of simulation dimensions at the initial state.

to $1 \times 10^{6} \mathrm{~W} / \mathrm{m}^{2} \mathrm{~K}$ may not be conducive to the extrusion of a thin metal plate from a chamber because solidification occurs before reaching the target region. The location of the interface strongly depends on heat transfer coefficients below $10^{4} \mathrm{~W} / \mathrm{m}^{2} \mathrm{~K}$. From these results, it is evident that the position of the solid-liquid interface can be controlled by the heat transfer coefficient, which is an important factor affecting the formation of a solid-liquid interface. There seems to be a little difference between the heat transfer coefficients of silicon and tin; however, the difference appears big because the heat transfer coefficients are expressed in the $y$-axis in a log scale.

In Figure 5, the mapping results of the initial state are shown. The Kelvin temperature scale was used for mapping. The mapping results of each heat extrusion direction and heat transfer coefficient are shown in Table 1. For both the side and up-and-down routes at $1.0 \times 10^{2} \mathrm{~W} / \mathrm{m}^{2} \mathrm{~K}$, the cooling has no effect on the formation of a solid-liquid interface in the final state. The lowest temperature region is higher than the melting point of silicon. Because of latent heat, the silicon needs a large amount of heat extraction in order to solidify. For the same amount of heat extraction, the side route has a tendency to cool faster than the up-and-down direction. The result of the $1.0 \times 10^{4} \mathrm{~W} / \mathrm{m}^{2} \mathrm{~K}$ up-and-down route shows that the atypical mapping results may be caused by the external velocity. The up-and-down cooling is not sufficient to cool the entire melt. Therefore, heat remains in the melt, and the temperature gradient resulting from it may cause defects in the final product. Moreover, irregular distribution of temperature also affects the microstructure.

\section{Conclusions}

The numerical simulation model for CSC was modified to analyze the solid-liquid interface behavior of silicon and tin. The final location of the interface was calculated and tracked using the front-tracking and sharp-interface method. We concluded that the heat transfer coefficient is an important factor that affects the formation of a solid-liquid interface. In addition, the heat transfer coefficient is also found to influence the shape of the interface, as determined through an investigation of the temperature mapping results. Therefore, the heat transfer coefficient is considered to be the main parameter in the fabrication of a thin metal plate. A more accurate simulation with 3D modeling is necessary for optimizing the conditions for a microstructure-controlled CSC process. 


\section{Acknowledgments}

This work was supported by the New \& Renewable Energy Technology Development Program of the Korea Institute of Energy Technology Evaluation and Planning (KETEP) Grant funded by the Korea Government Ministry of Knowledge Economy (2010T100100449). It was also supported by Korea Institute of Energy Research (no. GP2012-0002).

\section{References}

[1] D. P. Woorduff, The Solid-Liquid Interface, Cambridge University, New York, NY, USA, 1973.

[2] A. R. Ubbelohde, The Molten State of Matter, Wiley, New York, NY, USA, 1978.

[3] C. Lee, W. Yoon, S. Sin et al., "Numerical simulation of solid liquid interface behavior during continuous strip-casting process," Journal of Nanoscience and Nanotechnology, vol. 13, no. 54, pp. 3346-3349, 2013.

[4] H. Kim, J. Lee, C. Lee et al., "Study of the microstructures and lifetime of spin-cast silicon sheets for photovoltaics," Journal of Nanoscience and Nanotechnology, vol. 13, no. 55, pp. 3559-3563, 2013.

[5] R. P. Fedkiw, T. Aslam, B. Merriman, and S. Osher, "A nonoscillatory eulerian approach to interfaces in multimaterial flows (the Ghost Fluid Method)," Journal of Computational Physics, vol. 152, no. 2, pp. 457-492, 1999.

[6] G. Tryggvason, B. Bunner, A. Esmaeeli et al., "A front-tracking method for the computations of multiphase flow," Journal of Computational Physics, vol. 169, no. 2, pp. 708-759, 2001.

[7] H. S. Carslaw and C. J. Jaeger, Conduction of Heat in Solid, Clarendon Press, Oxford, UK, 1959.

[8] J. Glimm, M. J. Graham, J. Grove et al., "Front tracking in two and three dimensions," Computers and Mathematics with Applications, vol. 35, no. 7, pp. 1-11, 1998.

[9] S. Shin and D. Juric, "Modeling three-dimensional multiphase flow using a level contour reconstruction method for front tracking without connectivity," Journal of Computational Physics, vol. 180, no. 2, pp. 427-470, 2002.

[10] S. Shin, S. I. Abdel-Khalik, V. Daru, and D. Juric, "Accurate representation of surface tension using the level contour reconstruction method," Journal of Computational Physics, vol. 203, no. 2, pp. 493-516, 2005.

[11] N. Al-Rawahi and G. Tryggvason, "Numerical simulation of dendritic solidification with convection: two-dimensional geometry," Journal of Computational Physics, vol. 180, no. 2, pp. 471-496, 2002.

[12] S. Chen, D. B. Johnson, P. E. Raad, and D. Fadda, "The surface marker and microcell method," International Journal for Numerical Methods in Fluids, vol. 25, no. 7, pp. 749-778, 1997.

[13] F. H. Harlow and J. E. Welch, "Numerical calculation of timedependent viscous incompressible flow of fluid with free surface," Physics of Fluids, vol. 8, no. 12, pp. 2182-2189, 1965.

[14] S. Shin and D. Juric, "A hybrid interface method for threedimensional multiphase flows based on front tracking and level set techniques," International Journal for Numerical Methods in Fluids, vol. 60, no. 7, pp. 753-778, 2009.

[15] S. Shin and D. Juric, "Simulation of droplet impact on a solid surface using the level contour reconstruction method," Journal of Mechanical Science and Technology, vol. 23, no. 9, pp. 24342443, 2009. 

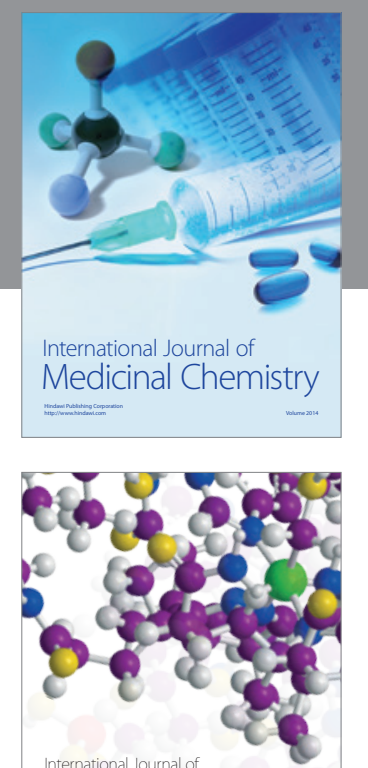

\section{Carbohydrate} Chemistry

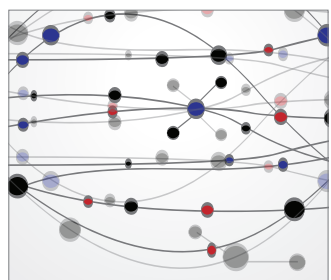

The Scientific World Journal
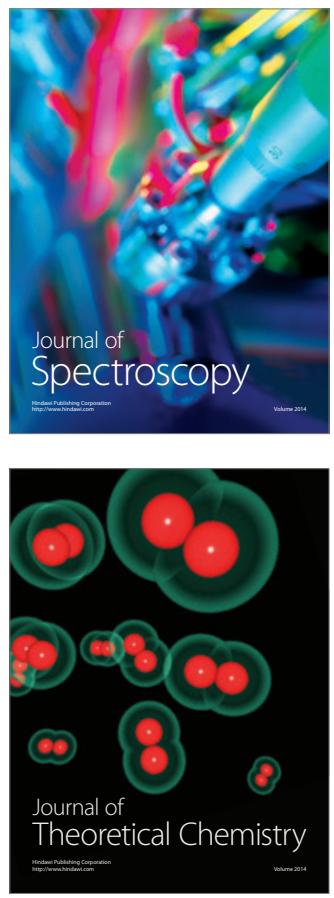
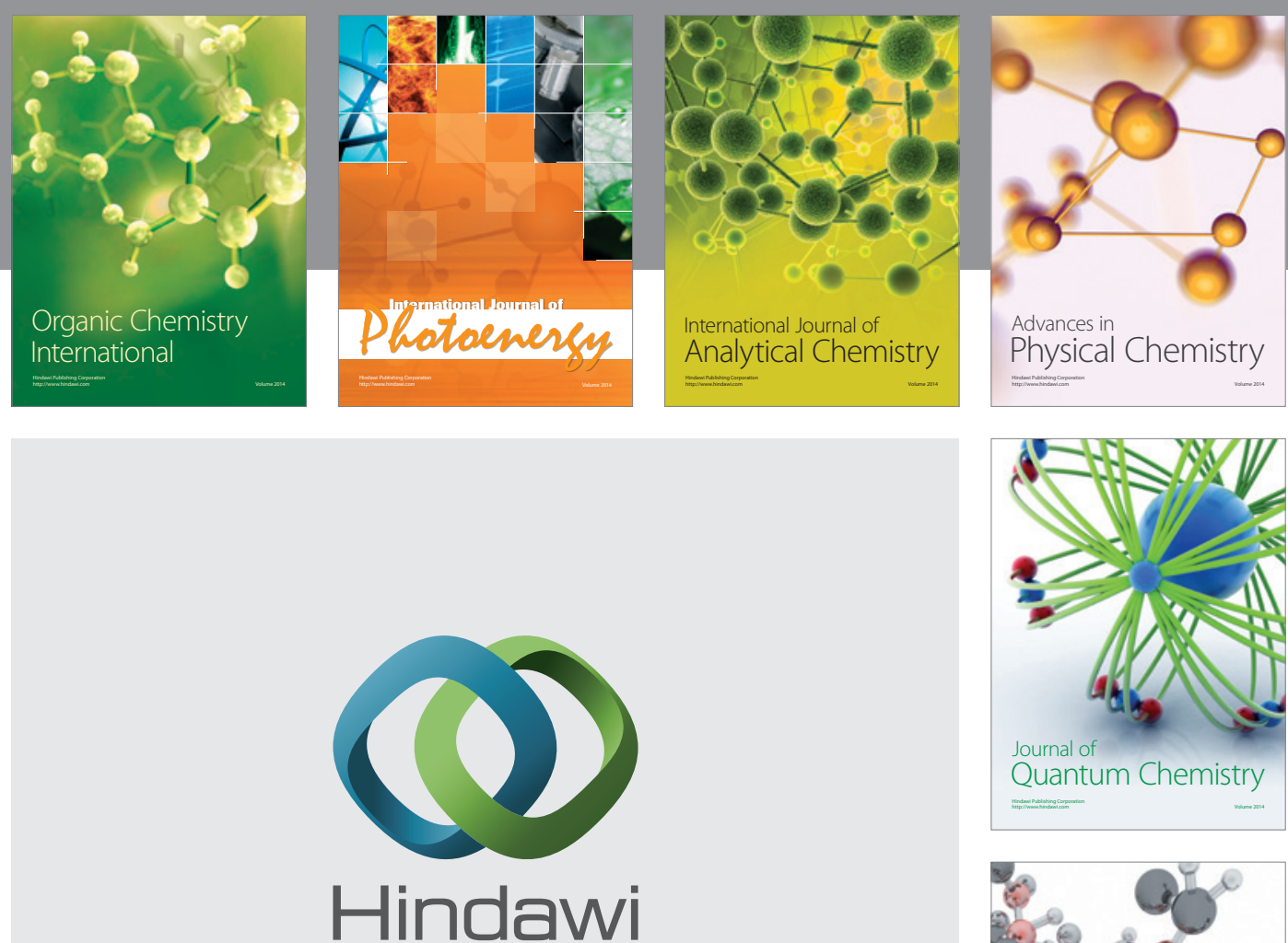

Submit your manuscripts at

http://www.hindawi.com

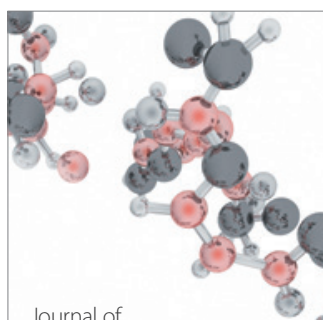

Analytical Methods

in Chemistry

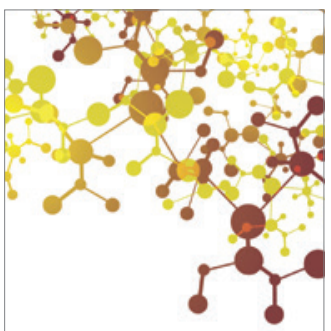

Journal of

Applied Chemistry

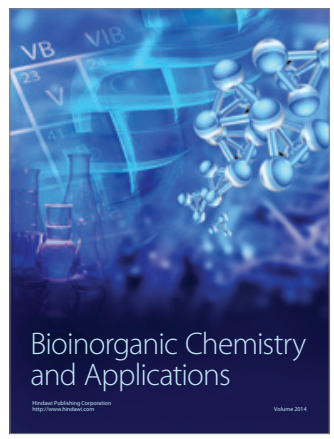

Inorganic Chemistry
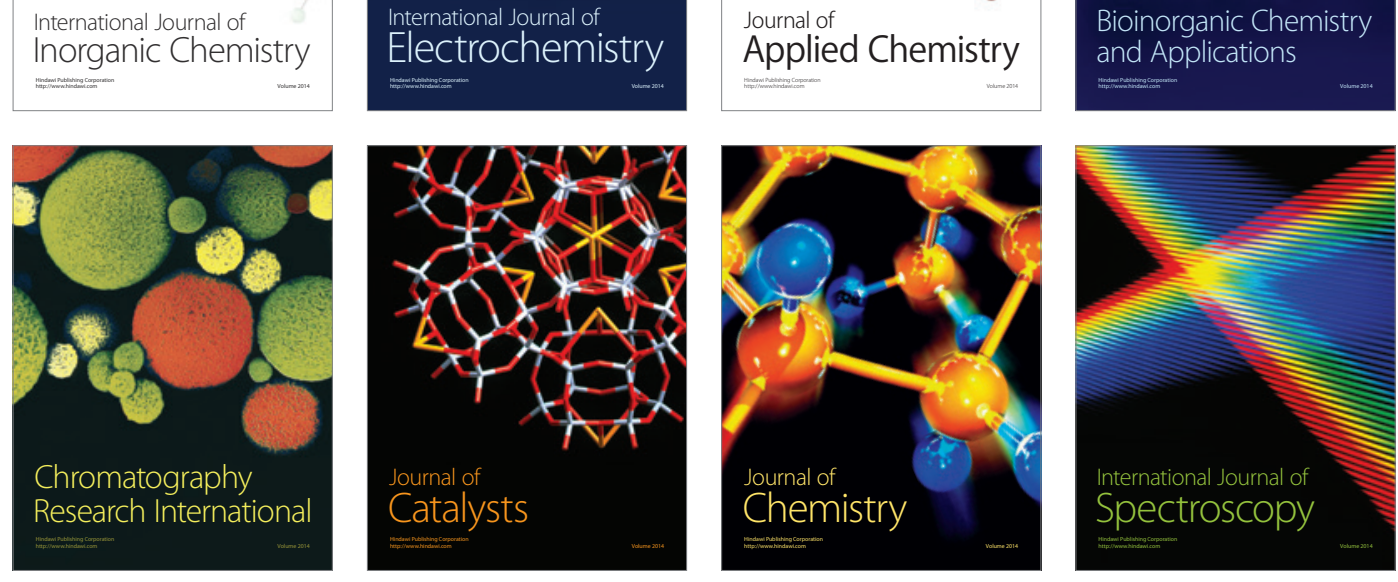